\title{
Surface magnetoinductive breathers in two-dimensional magnetic metamaterials
}

\author{
Maria Eleftheriou, ${ }^{1,2}$ Nikos Lazarides, ${ }^{3,4}$ George P. Tsironis, ${ }^{3}$ and Yuri S. Kivshar ${ }^{5}$ \\ ${ }^{1}$ Department of Materials Science and Technology, University of Crete, P.O. Box 2208, Heraklion, Crete 71003, Greece \\ ${ }^{2}$ Department of Music Technology and Acoustics, Technological Educational Institute of Crete, Rethymno, Crete 74100, Greece \\ ${ }^{3}$ Department of Physics, Institute of Electronic Structure and Laser Foundation for Research and Technology-Hellas, University of Crete, \\ P.O. Box 2208, Heraklion 71003, Greece \\ ${ }^{4}$ Department of Electrical Engineering, Technological Educational Institute of Crete, P.O. Box 140, Heraklion, Crete 71500, Greece \\ ${ }^{5}$ Nonlinear Physics Center, Research School of Physics and Engineering, Australian National University, Canberra, \\ Australian Capital Territory 0200, Australia
}

(Received 12 March 2009; published 9 July 2009)

\begin{abstract}
We study discrete surface breathers in two-dimensional lattices of inductively coupled split-ring resonators with capacitive nonlinearity. We consider both conservative (Hamiltonian) and analyze the properties of the modes localized in space and periodic in time (discrete breathers) located in the corners and on the edges of the lattice. We find that surface breathers in the Hamiltonian systems have lower energy than their bulk counterparts and they are generally more stable.
\end{abstract}

DOI: 10.1103/PhysRevE.80.017601

PACS number(s): 05.45.Yv, 63.20.Pw, 75.30.Kz, 78.20.Ci

Theoretical results on the existence of unusual types of discrete surface solitons localized in the corners or on the edges of two-dimensional (2D) photonic lattices [1-3] have been recently confirmed by the experimental observation of two-dimensional surface solitons in optically induced photonic lattices [4] and two-dimensional waveguide arrays laser written in fused silica $[5,6]$. These two-dimensional nonlinear surface modes demonstrate unusual features in comparison to their counterparts in truncated one-dimensional waveguide arrays [7-9]. In particular, in sharp contrast to onedimensional surface solitons, the mode threshold is lower at the surface than in the bulk making the mode excitation easier [2].

Recently, it was shown [10] that, similar to discrete solitons analyzed extensively for optical systems, surface discrete breathers can be excited near the edge of a onedimensional metamaterial created by a truncated array of nonlinear split-ring resonators. Networks of split-ring resonators (SRRs) that have nonlinear capacitive elements can support nonlinear localized modes or discrete breathers (DBs) under rather general conditions that depend primarily on the inductive coupling between SRRs and their resonant frequency $[11,12]$. The corresponding one-dimensional surface modes have somewhat lower energy (in the Hamiltonian case) and can easily be generated in one-dimensional SRR lattices [10].

In this Brief Report, we develop further those ideas and analyze two- dimensional lattices of split-ring resonators. Similar to the optical systems, we find that two-dimensional lattices of inductively coupled split-ring resonators with capacitive nonlinearity can support the existence of long-lived two-dimensional discrete breathers localized in the corners or on the edge of the lattice.

We consider a two-dimensional lattice of SRRs in both planar and planar-axial configurations [see Figs. 1(a) and 1(b)]. In the planar configuration, all SRR loops are in the same plane with their centers forming an orthogonal lattice, while in the planar-axial configuration the loops have planar arrangement in one direction and axial configuration in the other direction. Each SRR is equivalent to a nonlinear $R L C$ circuit, with Ohmic resistance $R$, self-inductance $L$, and capacitance $C$. We assume that the capacitor $C$ contains a nonlinear Kerr-type dielectric, so that the permittivity $\epsilon$ can be presented in the form

$$
\epsilon\left(|\mathbf{E}|^{2}\right)=\epsilon_{0}\left(\epsilon_{\ell}+\alpha \frac{|\mathbf{E}|^{2}}{E_{c}^{2}}\right),
$$

where $\mathbf{E}$ is the electric field with the characteristic value $E_{c}$, $\epsilon_{\ell}$ is the linear permittivity, $\epsilon_{0}$ is the permittivity of the vacuum, and $\alpha=+1(-1)$ corresponds to self-focusing (self-defocusing) nonlinearity, respectively. As a result, each SRR acquires the field-dependent capacitance $C\left(|\mathbf{E}|^{2}\right)=\epsilon\left(\left|\mathbf{E}_{g}\right|^{2}\right) A / d_{g}$, where $A$ is the area of the cross section of the SRR wire, $\mathbf{E}_{g}$ is the electric field induced along the SRR slit, and $d_{g}$ is the size of the slit. The field $\mathbf{E}_{g}$ is induced by the magnetic and/or the electric components of the applied electromagnetic field, depending on the relative orientation of the field with respect to the SRR plane and the slits [13]. Below we assume that the magnetic component of the incident (applied) electromagnetic field is always perpendicular to the SRR plane, so that the electric field component is transverse to the slit. With this assumption, only the magnetic component of the field excites an electromotive force in SRRs, resulting in an oscillating current in each SRR loop. This results in the development of an oscillating voltage dif-

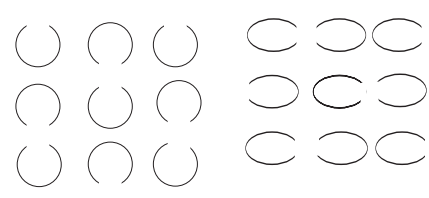

(a)

(b)

FIG. 1. Schematic of a two-dimensional lattice of split-ring resonators for (a) planar and (b) planar-axial geometries. In both the geometries the magnetic component of the applied field is directed along the SRR axes, while the electric field component is transverse to the slits. 
ference $U$ across the slits or, equivalently, of an oscillating electric field $\mathbf{E}_{g}$ in the slits.

If $Q$ is the charge stored in each SRR capacitor, from a general relation of a voltage-dependent capacitance $C(U)=d Q / d U$ and Eq. (1), we obtain

$$
Q=C_{\ell}\left(1+\alpha \frac{U^{2}}{3 \epsilon_{\ell} U_{c}^{2}}\right) U,
$$

where $U=d_{g} E_{g}, C_{\ell}=\epsilon_{0} \epsilon_{\ell}\left(A / d_{g}\right)$ is the linear capacitance, and $U_{c}=d_{g} E_{c}$. We assume that the arrays are placed in a timevarying and spatially uniform magnetic field of the form

$$
H=H_{0} \cos (\omega t),
$$

where $H_{0}$ is the field amplitude, $\omega$ is the field frequency, and $t$ is the time variable. The excited electromotive force $\mathcal{E}$, which is the same in all SRRs, is given by the expression

$$
\mathcal{E}=\mathcal{E}_{0} \sin (\omega t), \quad \mathcal{E}_{0} \equiv \mu_{0} \omega S H_{0},
$$

where $S$ is the area of each SRR loop and $\mu_{0}$ is the permittivity of vacuum. Each SRR exposed to the external field given by Eq. (3) is a nonlinear oscillator exhibiting a resonant magnetic response at a particular frequency, which is very close to its linear resonance frequency $\omega_{\ell}=1 / \sqrt{L C_{\ell}}$ (for $R \simeq 0$ ).

All SRRs in an array are coupled together due to magnetic dipole-dipole interaction through their mutual inductances. However, we assume below only nearest-neighbor interactions, so that the neighboring SRRs are coupled through their mutual inductances $M_{x}$ and $M_{y}$. This is a good approximation in the planar configurations [see Fig. 1(a)], even if SRRs are located very close. The validity of the nearest-neighbor approximation for the planar-axial configuration [see Fig. 1(b)] has been verified by taking into account the interaction of SRRs with their four nearest neighbors. Assuming that the mutual inductance $M_{x, y}^{(s)}$ between an SRR and its $s$-th neighbor decays with distance as $M_{x, y}^{(s)} \simeq M_{x, y} / s^{3}$ [12], we find practically the same results. Therefore, the electrical equivalent of an SRR array in an alternating magnetic field is an array of nonlinear $R L C$ oscillators coupled with their nearest neighbors through their mutual inductances; the latter are being driven by identical alternating voltage sources. Equations describing the dynamics of the charge $Q_{n, m}$ and the current $I_{n, m}$ circulating in the $(n, m)$ th SRR may be derived from Kirchhoff's voltage law for each SRR $[11,13]$,

$$
\begin{gathered}
\frac{d Q_{n, m}}{d t}=I_{n, m}, \\
L \frac{d I_{n, m}}{d t}+R I_{n, m}+f\left(Q_{n, m}\right)=-M_{x}\left(\frac{d I_{n-1, m}}{d t}+\frac{d I_{n+1, m}}{d t}\right) \\
-M_{y}\left(\frac{d I_{n, m-1}}{d t}+\frac{d I_{n, m+1}}{d t}\right)+\mathcal{E},
\end{gathered}
$$

where $f\left(Q_{n, m}\right)=U_{n, m}$ is given implicitly from Eq. (2). Using the relations

$$
\omega_{\ell}^{-2}=L C_{\ell}, \quad \tau=t \omega_{\ell}, \quad I_{c}=U_{c} \omega_{\ell} C_{\ell}, \quad Q_{c}=C_{\ell} U_{c},
$$

$$
\mathcal{E}=U_{c} \varepsilon, \quad I_{n, m}=I_{c} i_{n, m}, \quad Q_{n, m}=Q_{c} q_{n, m},
$$

and Eq. (4), we normalize Eqs. (5) and (6) to the form

$$
\begin{gathered}
\frac{d q_{n, m}}{d \tau}=i_{n, m}, \\
\frac{d i_{n, m}}{d \tau}+\gamma i_{n, m}+f\left(q_{n, m}\right)+\lambda_{x}\left(\frac{d i_{n-1, m}}{d \tau}+\frac{d i_{n+1, m}}{d \tau}\right) \\
+\lambda_{y}\left(\frac{d i_{n, m-1}}{d \tau}+\frac{d i_{n, m+1}}{d \tau}\right)=\varepsilon_{0} \sin (\Omega \tau),
\end{gathered}
$$

where $\gamma=R C_{\ell} \omega_{\ell}$ is the loss coefficient, $\lambda_{x, y}=M_{x, y} / L$ are the coupling parameters in the $x$ and the $y$ directions, respectively, and $\varepsilon_{0}=\mathcal{E}_{0} / U_{c}$. Note that the loss coefficient $\gamma$, which is usually small $(\gamma \ll 1)$, may account both for Ohmic and radiative losses [14]. Neglecting losses and without an applied field, Eqs. (9) and (10) can be derived from the Hamiltonian

$$
\mathcal{H}=\sum_{n, m}\left\{\frac{1}{2} \dot{q}_{n, m}^{2}+V_{n, m}\right\}-\sum_{n, m}\left\{\lambda_{x} \dot{q}_{n, m} \dot{q}_{n+1, m}+\lambda_{y} \dot{q}_{n, m} \dot{q}_{n, m+1}\right\},
$$

where the nonlinear on-site potential $V_{n, m}$ is given by

$$
V_{n, m} \equiv V\left(q_{n, m}\right)=\int_{0}^{q_{n, m}} f\left(q_{n, m}^{\prime}\right) d q_{n, m}^{\prime}
$$

and $\dot{q}_{n, m} \equiv d q_{n, m} / d \tau$. The analytical solution of Eq. (2) for $u_{n, m}=f\left(q_{n, m}\right)$ with the conditions of $u_{n, m}$ being real and $u_{n, m}\left(q_{n, m}=0\right)=0$ gives the approximate expression

$$
f\left(q_{n, m}\right) \simeq q_{n, m}-\frac{\alpha}{3 \epsilon_{\ell}} q_{n, m}^{3}+3\left(\frac{\alpha}{3 \epsilon_{\ell}}\right)^{2} q_{n, m}^{5},
$$

which is valid for relatively low $q_{n}\left(q_{n}<1, n=1,2, \ldots, N\right)$. Thus, the on-site potential is soft for $\alpha=+1$ and hard for $\alpha=-1$. In the $2 \mathrm{D}$ case the mutual inductances $M_{x}$ and $M_{y}$ may differ both in their sign, depending on the configuration, and their magnitude. Actually, even in the planar 2D configuration with $d_{x}=d_{y}$ a small anisotropy should be expected because we consider SRRs having only one slit. This anisotropy can be effectively taken into account by considering slightly different coupling parameters $\lambda_{x}$ and $\lambda_{y}$. The coupling parameters $\lambda_{x, y}$ as well as the loss coefficient $\gamma$ can be calculated numerically for this specific model with a high accuracy. However, for our purposes, it is sufficient to estimate these parameters for realistic (experimental) array parameters, ignoring the nonlinearity of the SRRs and the effects due to the weak coupling as in Refs. [11,12] with the following typical values $\lambda \approx 0.02$ and $\gamma \approx 0.01$.

We construct discrete breathers located in the corner of a two-dimensional lattice of $15 \times 15$ sites using the anticontinuous limit method as in Ref. [11] for the set of Eqs. (9) and (10), setting $\gamma=0$ and $\varepsilon_{0}=0$ (Hamiltonian discrete breathers). For the case of $\alpha=+1$ corresponding to selffocusing nonlinearity and period $T_{b}=6.69$, we may construct linearly stable breathers for parameters up to $\lambda_{x}=\lambda_{y}=0.029$. The breather stability has been checked through the Floquet 


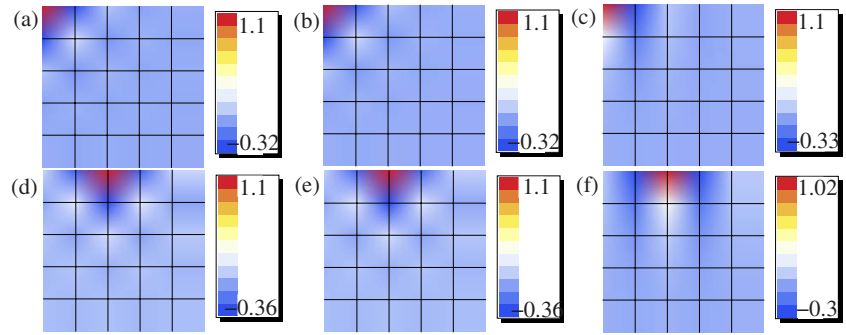

FIG. 2. (Color online) Density amplitudes $q_{n, m}$ for discrete Hamiltonian breathers constructed in (a)-(c) upper left corner or (d)-(f) upper edge of the lattice of $15 \times 15$ sites, $\alpha=+1$, and $T_{b}=6.69$. [(a),(c)] $\lambda_{x}=\lambda_{y}=0.028,[(\mathrm{~b}),(\mathrm{e})] \lambda_{x}=0.026$ and $\lambda_{y}=0.029$, and $[(\mathrm{c}),(\mathrm{f})] \lambda_{x}=0.029$ and $\lambda_{y}=-0.026$. All plots depict a $5 \times 5$ sublattice that includes the breather zones.

monodromy matrix throughout the Brief Report. For the case where an anisotropy is introduced, $\lambda_{x}<\lambda_{y}$, linearly stable discrete breathers can be constructed up to $\lambda_{x}=0.028$ and simultaneously $\lambda_{y}=0.031$, or for the case of planar-axial configuration up to $\lambda_{x}=0.031$ and at the same time $\lambda_{y}=-0.028$. If we look for discrete breathers constructed in the middle of the upper edge of the lattice, for example, we find that the values of the coupling where an instability occurs are slightly decreased (e.g., the upper stability limit of coupling for planar geometry is $\left.\lambda_{x}=\lambda_{y}=0.028\right)$. Several cases of linearly stable discrete breathers are shown in Fig. 2 for $\alpha=+1$. The same analysis holds for $\alpha=-1$ (defocusing nonlinearity) where the upper stability limit for the values of couplings are on the same order of magnitude as for $\alpha=+1$, both for the corner and the edge breathers (see Fig. 3). The breather period in the latter case is $T_{b}=5.8$.

Localized modes in the damped-driven case are constructed for $\gamma=0.01, \varepsilon_{0}=0.04$, and $\alpha=+1$ with the method described in Ref. [11]. The resulting localized modes are called dissipative breathers, and their examples are shown in Fig. 4 for $T_{b}=5.8$ and (a) $\lambda_{x}=\lambda_{y}=0.0007$, (b) $\lambda_{x}=0.0022$ and $\lambda_{y}=0.0052$, and (c) $\lambda_{x}=0.0052$ and $\lambda_{y}=-0.0022$. The dissipative modes have been evolved in time, and we found that at long times some dissipative breathers constructed for relatively large couplings loose their initial shape and finally decay.

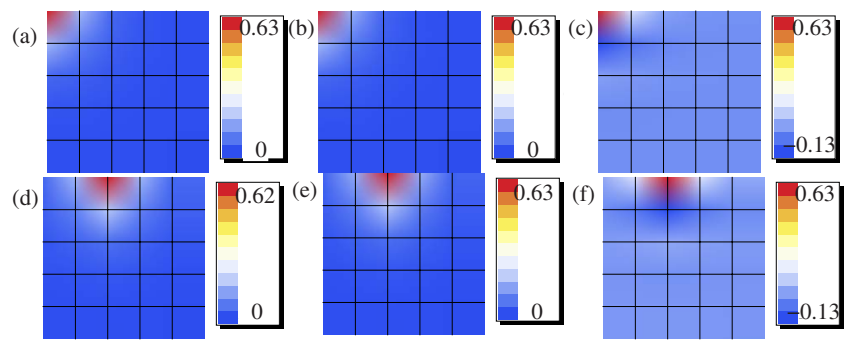

FIG. 3. (Color online) Density amplitude $q_{n, m}$ for discrete Hamiltonian breathers constructed in (a)-(c) upper left corner or in (d)-(f) upper edge of a lattice of $15 \times 15$ sites, $\alpha=-1$ and $T_{b}=5.8$. [(a),(d) $\lambda_{x}=\lambda_{y}=0.030,[(\mathrm{~b}),(\mathrm{e})] \quad \lambda_{x}=0.028$ and $\lambda_{y}=0.031$, and $[(\mathrm{c}),(\mathrm{f})] \lambda_{x}=0.028$ and $\lambda_{y}=-0.025$. All plots depict the $5 \times 5$ sublattice around the linearly stable breathers.
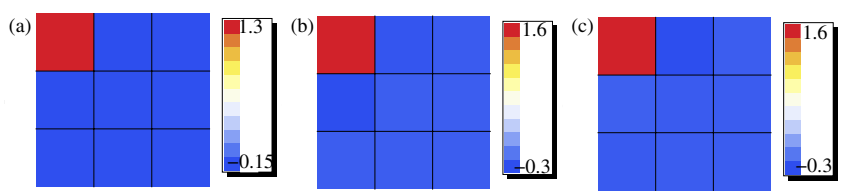

FIG. 4. (Color online) Density amplitude $q_{n, m}$ for discrete dissipative breathers constructed in the upper left corner of the lattice of $15 \times 15$ sites for $\gamma=0.01, \varepsilon_{0}=0.04, \alpha=+1$, and $T_{b}=6.82$ constructed in the upper left corner for (a) $\lambda_{x}=\lambda_{y}=0.0007$, (b) $\lambda_{x}$ $=0.0022$ and $\lambda_{y}=0.0052$, and (c) $\lambda_{x}=0.0052$ and $\lambda_{y}=-0.0022$. All plots depict the $3 \times 3$ sublattice around the breather. Dissipative breathers are very narrow and essentially confined on one lattice site.

Additionally, we calculate the total energy of discrete breathers in a lattice with planar and planar-axial configurations for $\alpha=+1$ and $T_{b}=6.69$ (Hamiltonian case). Figure 5 shows the energy histograms of the relevant corner of the lattice normalized to the energy of the corner $(1,1)$ breather. In order to construct the histograms centered in each of the lattice sites, we normalized it to the edge breather energy. In case (a) the discrete breather is constructed in a lattice of coupling $\lambda_{x}=\lambda_{y}=0.028$, in (b) the case with anisotropy in couplings $\lambda_{x}=0.026$ and $\lambda_{y}=0.029$, while in case (c) couplings are $\lambda_{x}=0.029$ and $\lambda_{y}=-0.026$. The energy of the discrete breathers as a function of the lattice site increases, i.e., as the discrete breather is constructed in the interior of the lattice, the energy is larger compared to the discrete breather that is located in the corner of the lattice.

We note that in the one-dimensional case the bulk breathers have lower energy compared to the surface ones [10], while in two-dimensional lattice the behavior is the contrary. We thus find that two-dimensional surface and especially edge breathers form easier.

Finally, we study the time evolution of the discrete breather that is constructed in the corner site $(1,1)$ and compare this case with a discrete breather centered at the $(3,3)$ site for the coupling $\lambda_{x}=\lambda_{y}=0.028$. The breather of the latter case after $t=95 T_{b}$ starts to loose its shape, in contrast to the breather of $(1,1)$ site which survives for much longer times, viz., $t=1450 T_{b}$ [see Fig. 6]. For different coupling values such as $\lambda_{x}=\lambda_{y}=0.01$ we find that both the corner $(1,1)$ and the inner $(3,3)$ breathers remain stable for at least $t=1450 T_{b}$. This feature, while compatible with the fact that

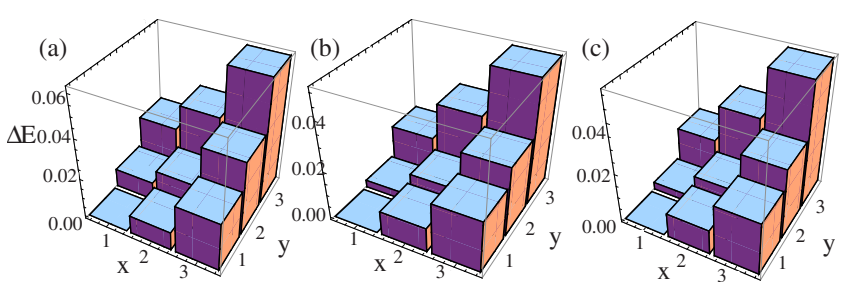

FIG. 5. (Color online) Histogram of the energy differences $\Delta E$ for a Hamiltonian breather with $\alpha=+1$ and $T_{b}=6.69$ constructed in the upper left $3 \times 3$ corner of the lattice. Case (a) $\lambda_{x}=\lambda_{y}=0.028$, case (b) $\lambda_{x}=0.026$ and $\lambda_{y}=0.029$, and case (c) $\lambda_{x}=0.029$ and $\lambda_{y}=-0.026$. To evaluate $\Delta E$ we calculate the energy of breathers centered in different sites and subtract the energy of the corner breather. 
(a)

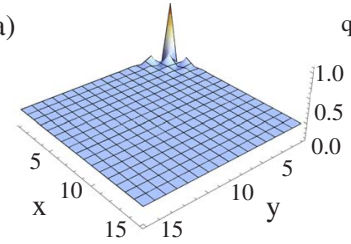

(c)

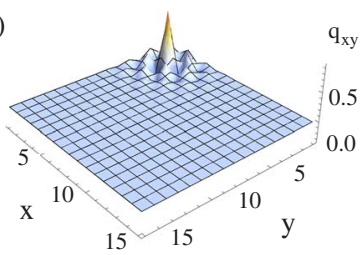

(b)

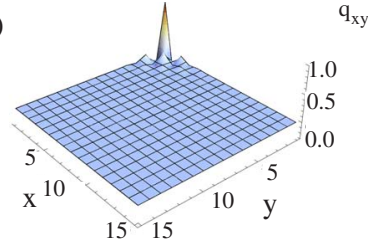

(d)

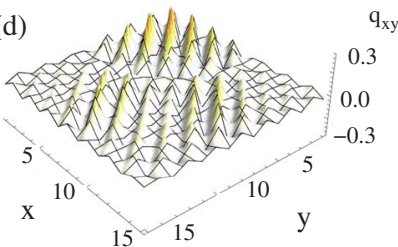

FIG. 6. (Color online) Amplitudes $q_{x, y}$ of the breather for $\alpha=+1, T_{b}=6.69$, and $\lambda_{x}=\lambda_{y}=0.028$ constructed on the site $(1,1)$ for (a) $t=0$ and (b) $t=1450 T_{b}$, and the breather constructed on site $(3,3)$ for (c) $t=0$ and (d) $t=1450 T_{b}$.

the corner breathers are more stable than the inner ones, shows additionally that in finite lattices small changes in parameters may affect the stability properties of the breathers [15].

In conclusion, we have studied surface discrete breathers located in the corner and on the edge of the two-dimensional lattices of the split-ring resonators. Using standard numerical methods, we found nonlinear localized modes both in the conservative and the dissipative systems. Two-dimensional breathers in conservative lattices have been found to be linearly stable for up to certain (large) values of the coupling coefficient, in both planar and planar-axial configurations of the split-ring-resonator lattices. Dissipative discrete surface breathers can retain their shapes for several periods of breather, and they depend critically on the lattice coupling. Finally, we found that discrete breathers located deep inside the lattice have higher energy compared to breathers located in corners and on the edges. This distinct two-dimensional feature of nonlinear localized modes contrasts with the onedimensional behavior being attributed to the larger number of neighbors of the two-dimensional lattice. Furthermore, the two-dimensional breathers located inside the lattice loose rapidly their initial shapes as they evolve in time, while the surface breathers are seen to be stable at least for $t \approx 1500 T_{b}$.
[1] K. G. Makris, J. Hudock, D. N. Christodoulides, G. Stegeman, O. Manela, and M. Segev, Opt. Lett. 31, 2774 (2006).

[2] R. A. Vicencio, S. Flach, M. I. Molina, and Yu. S. Kivshar, Phys. Lett. A 364, 274 (2007).

[3] H. Susanto, P. G. Kevrekidis, B. A. Malomed, R. CarreteroGonzález, and D. J. Frantzeskakis, Phys. Rev. E 75, 056605 (2007).

[4] X. Wang, A. Bezryadina, Z. Chen, K. G. Makris, D. N. Christodoulides, and G. I. Stegeman, Phys. Rev. Lett. 98, 123903 (2007).

[5] A. Szameit, Y. V. Kartashov, F. Dreisow, T. Pertsch, S. Nolte, A. Tünnermann, and L. Torner, Phys. Rev. Lett. 98, 173903 (2007).

[6] A. Szameit, Y. V. Kartashov, V. A. Vysloukh, M. Heinrich, F. Dreisow, T. Pertsch, S. Nolte, A. Tünnermann, F. Lederer, and L. Torner, Opt. Lett. 33, 1542 (2008).

[7] K. G. Makris, S. Suntsov, D. N. Christodoulides, G. I. Stegeman, and A. Haché, Opt. Lett. 30, 2466 (2005).

[8] S. Suntsov, K. G. Makris, D. N. Christodoulides, G. I. Stege- man, A. Haché, R. Morandotti, H. Yang, G. Salamo, and M. Sorel, Phys. Rev. Lett. 96, 063901 (2006).

[9] M. Molina, R. Vicencio, and Yu. S. Kivshar, Opt. Lett. 31, 1693 (2006).

[10] N. Lazarides, G. P. Tsironis, and Yu. S. Kivshar, Phys. Rev. E 77, 065601(R) (2008).

[11] N. Lazarides, M. Eleftheriou, and G. P. Tsironis, Phys. Rev. Lett. 97, 157406 (2006).

[12] M. Eleftheriou, N. Lazarides, and G. P. Tsironis, Phys. Rev. E 77, 036608 (2008).

[13] A. A. Zharov, I. V. Shadrivov, and Y. S. Kivshar, Phys. Rev. Lett. 91, 037401 (2003); I. V. Shadrivov, A. A. Zharov, N. A. Zharova, and Y. S. Kivshar, Photonics Nanostruct. Fundam. Appl. 4, 69 (2006).

[14] I. Kourakis, N. Lazarides, and G. P. Tsironis, Phys. Rev. E 75, 067601 (2007).

[15] A. M. Morgante, M. Johansson, S. Aubry, and G. Kopidakis, J. Phys. A 35, 4999 (2002). 